\title{
Risk and characteristics of tuberculosis after anti-tumor necrosis factor therapy for inflammatory bowel disease: a hospital-based cohort study from Korea
}

Jae Yong Lee ${ }^{1}$, Kyunghwan Oh'1, Hee Seung Hong ${ }^{1}$, Kyuwon Kim¹', Seung Wook Hong ${ }^{1}$, Jin Hwa Park', Sung Wook Hwang ${ }^{1}$, Dong-Hoon Yang ${ }^{1}$, Byong Duk Ye ${ }^{1}$, Jeong-Sik Byeon'1, Seung-Jae Myung ${ }^{1}$, Suk-Kyun Yang ${ }^{1}$, Ho-Su Lee ${ }^{2}$, Kyung-Wook Jo $\mathrm{O}^{3^{*+}}$ and Sang Hyoung Park ${ }^{*^{*}+}$

\begin{abstract}
Background: Anti-tumor necrosis factor (TNF) treatment for inflammatory bowel disease (IBD) increases the risk of tuberculosis (TB) infection. In the present study, we analyzed the clinical characteristics and risks of TB in Korean patients with IBD who received anti-TNF treatment.

Methods: The study included patients with IBD who were treated using anti-TNF agents between January 2001 and June 2018 at the Asan Medical Center. Overall, 1434 patients with ulcerative colitis or Crohn's disease were enrolled. We calculated the incidence of active TB infection after anti-TNF treatment and compared the clinical characteristics of the TB group with those of the non-TB group.

Results: Twenty-one patients (1.46\%) developed active TB infection, and the incidence rate of active TB was 366.73 per 100,000 person-years. In total, 198 patients (14.9\%) were positive for latent tuberculosis infection (LTBI), of whom only eight (4\%) did not complete LTBI treatment. The age at which the anti-TNF therapy was started was significantly higher in the TB group than in the non-TB group (HR 1.041,95\% Cl 1.014-1.069, $p=0.002$ ), and as age increased, so did the incidence rate of active TB infection (linearity $p<0.001$ ). There was no significant difference in the incidence rate of LTBI between the TB and non-TB groups (HR 0.896, 95\% Cl 0.262-3.066, $p=0.862$ ).
\end{abstract}

Conclusions: In patients with IBD, the incidence rate of TB increased with age at anti-TNF therapy initiation. Active treatment of LTBI may lower the incidence of TB in patients with IBD who are to undergo anti-TNF therapy.

Keywords: Inflammatory bowel diseases, Tuberculosis, Tumor necrosis factor inhibitor, Latent tuberculosis, Risk factors

*Correspondence: heathcliff6800@hanmail.net; umdalpin@hanmail.net ${ }^{\dagger}$ Kyung-Wook Jo and Sang Hyoung Park contributed equally as correspondence authors

1 Department of Gastroenterology, Asan Medical Center, University of Ulsan College of Medicine, 88 Olympic-ro 43-gil, Songpa-gu, Seoul 05505, Korea

${ }^{3}$ Department of Pulmonary and Critical Care Medicine, Asan Medical Center, University of Ulsan College of Medicine, 88 Olympic-ro 43-gil, Songpa-gu, Seoul 05505, Korea

Full list of author information is available at the end of the article

\begin{abstract}
Background
Inflammatory bowel disease (IBD) is a disorder involving abnormal chronic inflammation in the gastrointestinal tract, which is caused by immune dysregulation. The disorder encompasses ulcerative colitis (UC) and Crohn's disease (CD) $[1,2]$. The number of patients with IBD has increased recently in Korea and other Asian countries, where the disorder was once considered rare
\end{abstract}


[3-7]. Immune dysregulation in IBD results in overproduction of tumor necrosis factor (TNF)- $\alpha$, and monoclonal antibodies targeting TNF- $\alpha$ can suppress the abnormal immune response in IBD [8]. Such anti-TNF therapy has proven effective in both induction and maintenance therapy in patients with IBD, so the use of the anti-TNF therapy is increasing worldwide [9-11].

However, anti-TNF therapy increases the risk of infection [12, 13]. Tuberculosis (TB) is one infection associated with anti-TNF therapy, which is thought to inhibit the formation of granuloma and thus prevent the suppression of TB activation [14]. Because anti-TNF therapy increases the risk of active TB development through the reactivation of latent TB $[15,16]$, guidelines recommend diagnosing and treating latent tuberculosis infection (LTBI) before starting anti-TNF therapy $[12,16,17]$. Although the incidence of TB is decreasing worldwide, interest in the disease is increasing in Korea, which still has a higher incidence of TB and LTBI than more developed countries [18-20].

Several studies have investigated the development of TB after anti-TNF therapy in patients with IBD [14, 18, 21, 22]. However, most such research was conducted in multi-institution cohort studies, in which each institution often applied different standards of diagnosis, treatment, and follow-up. Therefore, it is worthwhile to analyze the risks and characteristics of TB infection in patients who have undergone standardized management of IBD and
LTBI before anti-TNF therapy in Korea. In the present study, we used a well-established hospital-based cohort in Korea to evaluate the incidence, characteristics, and risk factors of TB in patients with IBD who received antiTNF therapy.

\section{Methods \\ Study design}

The present retrospective study was conducted at the Asan Medical Center, a tertiary medical center in Korea. Between January 2001 and June 2018, 1811 patients with IBD (1386 with CD and 425 with UC) were prescribed anti-TNF agents in our institution. During the study period, the anti-TNF agents used to treat IBD included infliximab, adalimumab, and golimumab. According to the following criteria, 377 patients were excluded: (1) citizenship of a state other than Korea, (2) duration of follow-up or anti-TNF therapy $<1$ month, and (3) previous history of anti-TNF therapy at other medical centers. Consequently, 1434 patients with IBD were eligible for the analysis (Fig. 1).

The following patient information was collected from the medical records: age, sex, type of IBD, age at IBD diagnosis, smoking status, results of LTBI screening, history of TB, anti-TNF agent type, age at the start of anti-TNF therapy, and concomitant medication, such as steroids and immunomodulators. The study protocol

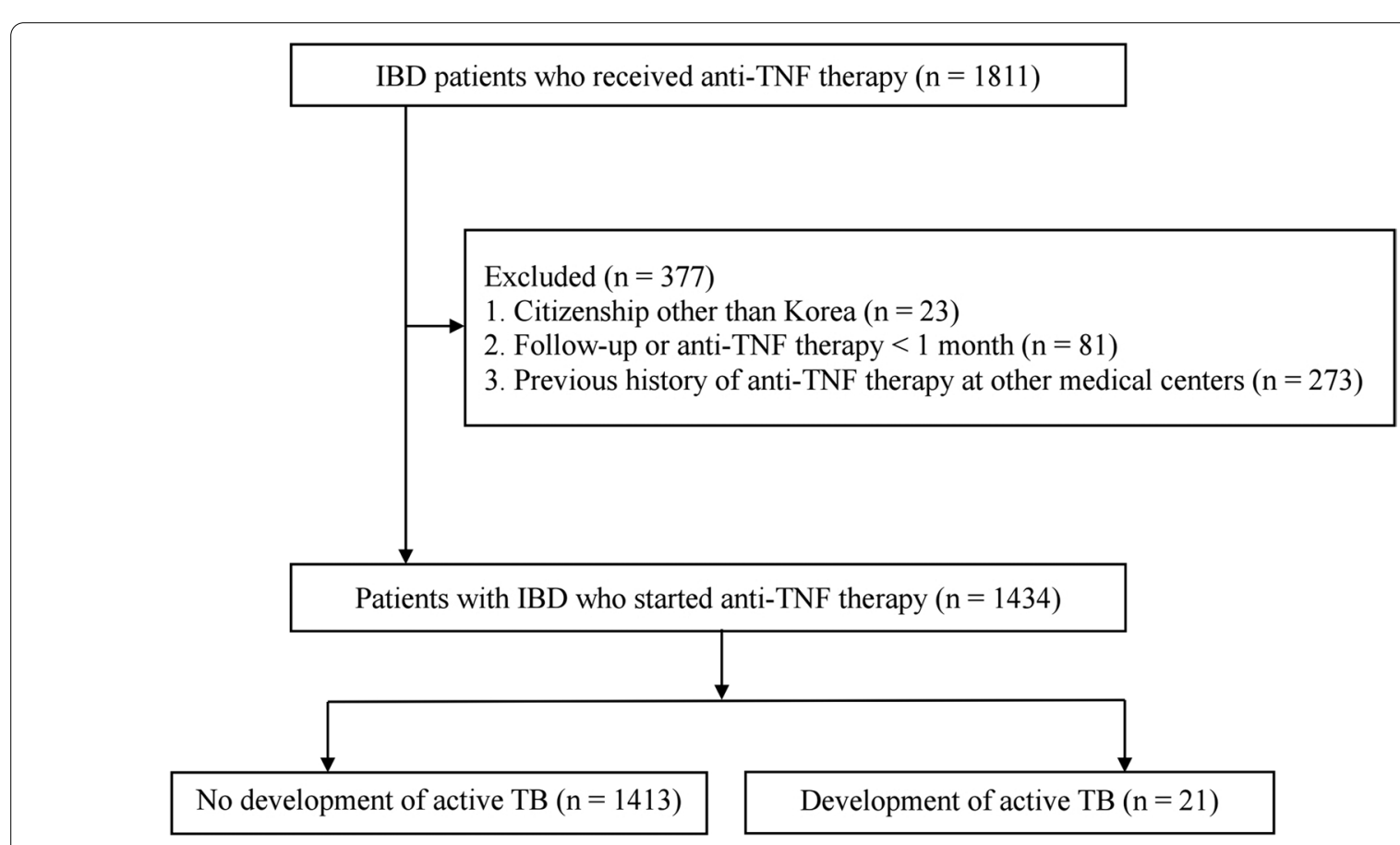

Fig. 1 Study flow diagram. IBD, inflammatory bowel disease; TNF, tumor necrosis factor; TB, tuberculosis 
was approved by the Institutional Review Board of Asan Medical Center (IRB No. 2018-1178).

\section{Screening and management of LTBI}

According to Korean guidelines, screening for LTBI is recommended before the initiation of anti-TNF therapy [23]. Most patients subject to LTBI screening were referred to TB specialists for work-up and management. They were checked for past medical history of TB infection and any symptoms suggestive of TB. LTBI tests consisted of simple chest X-rays, the tuberculin skin test (TST), and/or the interferon gamma release assay (IGRA). The TST was carried out following the Mendel-Mantoux method using purified protein derivative (PPD). An induration with a diameter of $\geq 10 \mathrm{~mm}$ was considered positive 48-72 $\mathrm{h}$ after PPD inoculation on the forearm [24]. The IGRA was performed using QuantiFERON $^{\circledR}$-TB Gold In-Tube (QFT-GIT; Cellestis, Carnegie, VIC, Australia) and/or T-SPOT ${ }^{\circledR}$.TB (Oxford Immunotec, Abingdon, UK). The test results were defined according to the manufacturer's instructions. The subjects were considered to have LTBI based on the following criteria: (1) patients with a positive TST or IGRA test in the absence of clinical or radiological signs of active TB or (2) those with no history of TB treatment and lesions suggesting spontaneously healed TB on chest $\mathrm{X}$-ray $[23,24]$.

Patients with a positive LTBI underwent TB prophylaxis, which usually started with isoniazid and rifampin for 3 months. If the patients had any comorbid disease that contraindicated this combination therapy, or if adverse effects occurred, rifampin for 4 months or isoniazid for 9 months was administered. Patients who completed LTBI screening were followed up during their anti-TNF therapy, regardless of whether they were positive for LTBI. At regular intervals, chest radiography was carried out, and the patients were checked for symptoms or signs suggesting TB.

\section{Statistical analysis}

Hazard ratios (HRs) and 95\% confidence intervals (CIs) were calculated using Cox proportional hazards models to evaluate the risk factors for TB development. The incidence rate (IR) of TB among patients with IBD was calculated as the number of patients with TB per 100,000 person-years of the population. The $95 \%$ CI of the IR was based on the Poisson probabilities of the corresponding patients, and the linearity $p$ value of IR was calculated based on the log-binomial model. The TB-free survival probability was constructed using the Kaplan-Meier method. All $p$ values $<0.05$ were considered statistically significant. Statistical analyses were performed using the
R program, version 4.0.3 (The R Foundation for Statistical Computing, Vienna, Austria).

\section{Results}

Baseline characteristics of the study patients

A total of 1434 patients with IBD who started anti-TNF therapy were included. The baseline characteristics of the patients who underwent anti-TNF therapy are shown in Table 1. The mean age at anti-TNF therapy initiation was $31.35 \pm 13.59$ years, and the mean follow-up period was 48.54 months. Among the patients, 940 (65.6\%) were male and $1102(76.8 \%)$ had CD. There were 991 (69.1\%) non-smokers. Three types of anti-TNF agents were used: infliximab, adalimumab, and golimumab. There were 1259 patients $(87.8 \%)$ who were given only one agent, and most of them used infliximab. There were 166 patients $(11.6 \%)$ who used two drugs and three $(0.2 \%)$ who used three drugs.

With regard to the tests for LTBI, 939 patients underwent the TST and 1328 were tested using the IGRA. There were 966 patients who underwent both the IGRA and the TST. In total, 1333 patients (93.0\%) completed LTBI screening, of whom 198 (14.9\%) were positive for

Table 1 Baseline characteristics of 1434 patients who underwent anti-TNF therapy

\begin{tabular}{ll}
\hline Characteristics & \\
\hline Age of IBD diagnosis (years) & $25.81 \pm 12.45$ \\
Age at anti-TNF therapy initiation (years) & $31.35 \pm 13.59$ \\
Follow up (months) & $48.54 \pm 36.48$ \\
Sex (male:female) & $940: 494$ \\
IBD type (UC:CD) & $332(23.2 \%): 1102(76.8 \%)$ \\
History of smoking (no:yes) & $991(69.1 \%): 443(30.9 \%)$ \\
Anti-TNF agents & \\
One & $1259(87.8 \%)$ \\
Infliximab & $939(65.5 \%)$ \\
$\quad$ Adalimumab & $311(21.7 \%)$ \\
$\quad$ Golimumab & $9(0.6 \%)$ \\
Two & $172(12.0 \%)$ \\
Infliximab, Adalimumab & $166(11.6 \%)$ \\
Infliximab, Golimumab & $2(0.1 \%)$ \\
$\quad$ Adalimumab, Golimumab & $4(0.3 \%)$ \\
Three & $3(0.2 \%)$ \\
LTBI diagnosis & $1333(93.0 \%)$ \\
Done & $1135(85.1 \%)$ \\
Negative & $198(14.9 \%)$ \\
Positive & $190(96 \%)$ \\
Completion of TB prophylaxis & $8(4.0 \%)$ \\
Failure to complete TB prophylaxis & $101(7.0 \%)$ \\
Not done, other & \\
\hline
\end{tabular}

TNF, tumor necrosis factor; IBD, inflammatory bowel disease; UC, ulcerative colitis; $C D$, Crohn's disease; LTBI, latent tuberculosis infection; TB, tuberculosis 
LTBI. Among them, 166 patients (83.8\%) underwent TB prophylaxis according to our protocol. Twenty-four patients (12.1\%) had a history of TB medication for active TB or to identify intestinal TB and did not require additional TB prophylaxis. Only 8 patients $(4.0 \%)$ did not complete TB prophylaxis.

\section{Clinical characteristics of patients who developed active TB during anti-TNF therapy}

Twenty-one patients with IBD were diagnosed with TB during anti-TNF therapy. The proportion of active TB development was $1.46 \%$ and the incidence rate was 366.73 per 100,000 person-years. The baseline characteristics of the patients who developed TB are described in Table 2. There were six patients with UC and 15 with $\mathrm{CD}$, and there were 12 male and nine female patients. The median duration from the start of anti-TNF therapy to the diagnosis of active TB infection was 14 months (range 1-95). One patient had been diagnosed and treated for TB in the past. Seventeen patients developed TB while using infliximab. Four patients diagnosed with TB had changed to adalimumab or had received adalimumab from the beginning. Three of the patients with TB had positive LTBI screening, and had completed TB prophylaxis. Fourteen of the patients with TB received other immunosuppressants including steroids, azathioprine, and methotrexate within 3 months prior to $\mathrm{TB}$ diagnosis. Diabetes, chronic renal failure, and acquired immunodeficiency syndrome, which can influence the morbidity of TB, were not identified in any of the patients with TB. There were 18 cases $(85.7 \%)$ of TB involving the lung and eight (38.1\%) of TB involving other organs. In 20 patients, the TB was completely cured, while one patient was undergoing treatment at the end of the study.

\section{Comparison between TB and non-TB groups}

We used the Cox proportional hazard model to identify the risk of TB development in patients with IBD treated using anti-TNF therapy (Table 3). The mean age at which anti-TNF therapy was started was higher in the TB group $(\mathrm{n}=21)$ than in the non-TB group $(\mathrm{n}=1413 ; 39.57 \mathrm{vs}$. 31.22 years; $p=0.002$ ). There were no significant differences between the two groups in sex, IBD type, smoking status, TB infection history and screening tests for LTBI. In patients who underwent LTBI screening, the positive rates of LTBI did not differ between the two groups $(14.3 \%$ vs. $13.8 \% ; p=0.862)$. There were no significant differences in the incidence of TB between the LTBI-positive and LTBI-negative groups $(p=0.86)$.

\section{Incidence rate of TB development according to age}

The study subjects were divided into four age groups $(0-19,20-39,40-59$, and $\geq 60$ years $)$; the incidence rate of TB in each group was checked and cross-analysis was performed (Fig. 2). The incidence rate of TB in each age group was $0,323.4,756.6$, and 1557.8 per 100,000 person-years, respectively, and the incidence rate increased linearly as the age increased (linearity $p$ value $<0.001$ ). The incidence of TB in LTBI-negative patients $(\mathrm{n}=1135)$ was $0,300,915.6$, and 2931.6 per 100,000 person-years, respectively (linearity $p=0.011$ ), and the incidence of TB in patients receiving only infliximab $(\mathrm{n}=939)$ was 0 , 359.6, 798.7, and 2061.9 per 100,000 person-years, respectively (linearity $p$ value $<0.001$ ).

\section{Discussion}

In Korea, the incidence of IBD is gradually increasing in all age groups, including elderly citizens [25]. Furthermore, the age of patients with IBD who are receiving treatment is increasing over time. Because of this trend, there is growing research interest in medication and its associated morbidities in older patients with IBD. As age increases, the production of $\mathrm{T}$ cells and the $\mathrm{T}$ cellmediated response decreases, suppressing the formation of granulomas by mononuclear phagocytes activated by Mycobacterium tuberculosis infection and thus increasing the risk of active TB infection [26, 27]. The prevalence of nutritional deficiencies and other age-related diseases also increases, which can influence the morbidity of TB $[27,28]$. In the present study, we identified a significant age difference between the TB group and the non-TB group of patients with IBD (39.57 vs. 31.22 years; $p=0.002$ ), and there was a linear increase in the incidence rate of TB infection as the age at anti-TNF therapy initiation increased (Fig. 2).

It can be assumed that the age at anti-TNF therapy initiation is related to the incidence of TB in patients with IBD because of the high prevalence of LTBI-positivity in Korea in the older age group [23]. However, the incidence rate of TB development in LTBI-negative patients $(n=1135)$ is also higher in the older age group. It follows that age itself may be a risk factor for TB development in patients with IBD undergoing anti-TNF therapy, regardless of LTBI. In the infliximab-alone group, which contained the highest number of patients among the various anti-TNF therapy groups, the incidence rate increased linearly as the age group increased. However, in patients receiving other single or multiple anti-TNF agents, there was small sample size, so the prevalence of TB must be carefully interpreted with each anti-TNF agent. In addition, the present study was carried out in a country with an intermediate TB burden [23, 29]; therefore, caution should be exercised when applying the results to regions with different incidence rates.

In the present study, the incidence rate of TB development in patients with IBD treated with anti-TNF therapy 


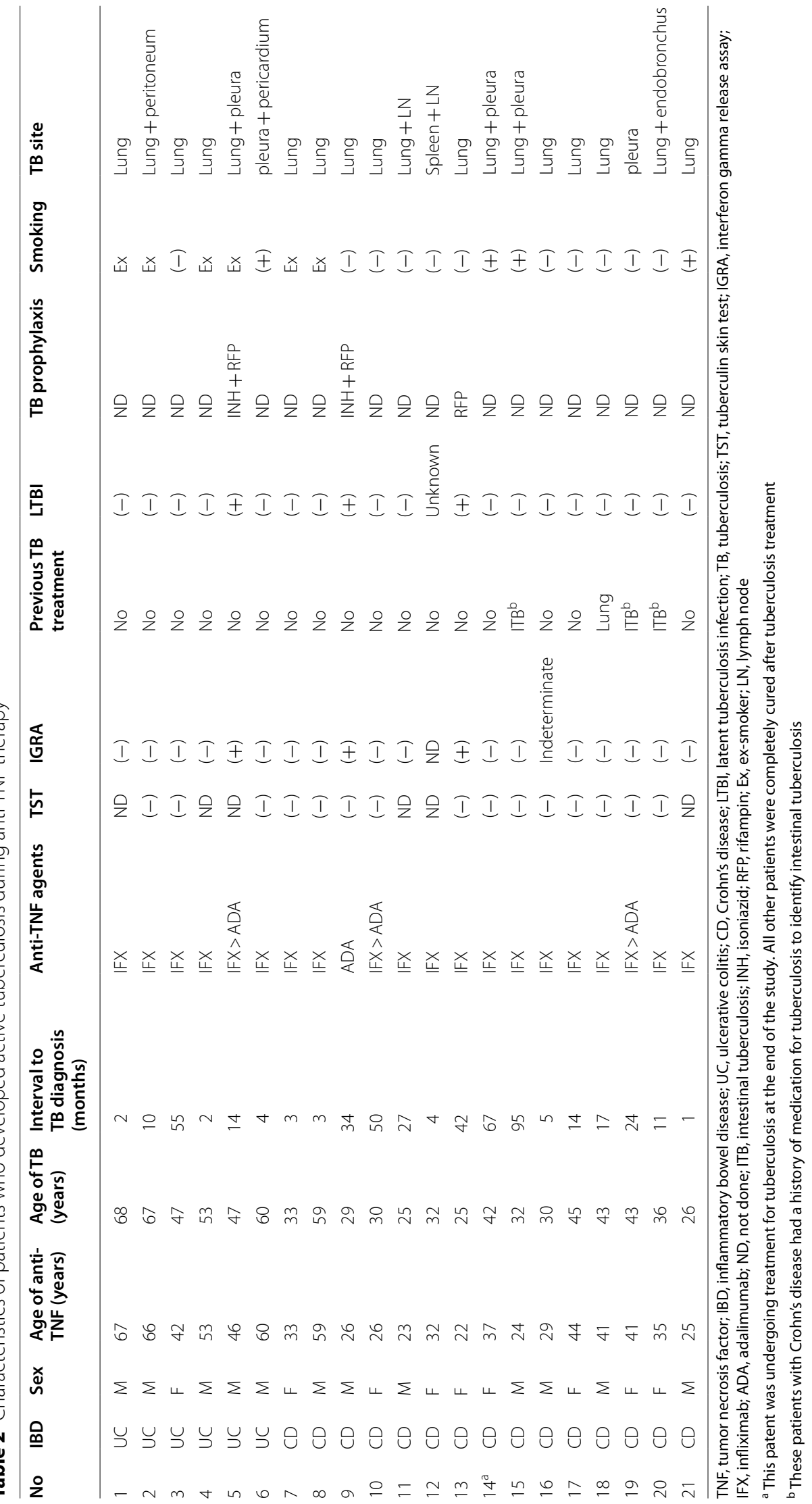


Table 3 Risks of active tuberculosis infection during anti-TNF therapy, derived from a Cox proportional hazards model

\begin{tabular}{|c|c|c|c|c|c|}
\hline & $\begin{array}{l}\text { TB }+ \text { group } \\
(\mathrm{n}=21)\end{array}$ & & $\begin{array}{l}\text { TB- group } \\
(n=1413)\end{array}$ & $\begin{array}{l}\text { Hazard ratio } \\
(95 \% \mathrm{Cl})\end{array}$ & $p$ value \\
\hline Age at anti-TNF (years) & 39.57 & 31.22 & & $1.041(1.014-1.069)$ & 0.002 \\
\hline Age of IBD diagnosis (years) & 32.81 & 25.70 & & $1.039(1.012-1.067)$ & 0.005 \\
\hline Female & $9(42.9)$ & $485(34.3)$ & & $1.286(0.541-3.055)$ & 0.570 \\
\hline IBD type & & & & $0.660(0.254-1.714)$ & 0.393 \\
\hline UC & $6(28.6)$ & $323(23.1)$ & & & \\
\hline$C D$ & $15(71.4)$ & $1087(76.9)$ & & & \\
\hline History of smoking & $10(47.6)$ & $433(30.6)$ & & $2.094(0.889-4.932)$ & 0.091 \\
\hline Previous TB history & $1(4.7)$ & $48(3.4)$ & & $1.297(0.174-9.665)$ & 0.800 \\
\hline Screening for LTBI & & & & $1.170(0.417-3.284)$ & 0.765 \\
\hline IGRA + TST & $15(71.4)$ & $951(67.3)$ & & & \\
\hline IGRA & $5(23.8)$ & $361(25.5)$ & & & \\
\hline LTBI & $3(14.3)$ & $195(13.8)$ & & $0.896(0.262-3.066)$ & 0.862 \\
\hline
\end{tabular}

TNF, tumor necrosis factor; IBD, inflammatory bowel disease; UC, ulcerative colitis; CD, Crohn's disease; LTBI, latent tuberculosis infection; TB, tuberculosis

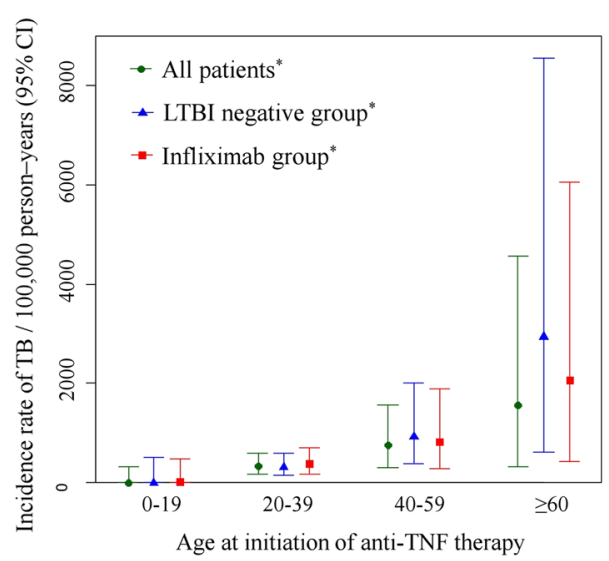

Fig. 2 Incidence rates (per 100,000 person-years) of active tuberculosis infection according to age: all patients (circle), latent tuberculosis-negative group (triangle), infliximab group (square). *Linearity $p<0.05$. TNF, tumor necrosis factor; TB, tuberculosis; LTBI, latent tuberculosis infection

was 366.73 per 100,000 person-years, and the prevalence was $1.46 \%$. In another multicenter, retrospective study carried out in Korea, 16 cases of TB diagnosis were reported among 376 patients with IBD who underwent anti-TNF therapy (incidence rate 1997.4 per 100,000 person-years) [18]. Similarly, in a study that analyzed data from the National Health Insurance system in Korea, the incidence rate was 554.1 per 100,000 person-years [30]. As such, the incidence rate of TB development in the present study was lower than that in other studies. In the present study, most patients with IBD in whom anti-TNF therapy was planned were referred to TB specialists for LTBI screening and management, as well as continued follow-up observation. As a result, a high rate of LTBI evaluation (93\%) was seen, and only eight out of the 198 patients did not complete TB prophylaxis, resulting in a high LTBI treatment completion rate (96\%). In the aforementioned study, on the other hand, among the 30 LTBI-positive patients, 16 (53\%) underwent TB prophylaxis [18]. Therefore, we believe that completion of LTBI treatment in LTBI-positive patients will lower the incidence rate and prevalence of TB development, and that a system for LTBI screening and management should be implemented to this end.

Another interesting finding was that the LTBI-positive rate did not show a significant difference between the TB and non-TB groups. Treatment of LTBI prior to immunosuppressant administration is thought to prevent active TB infection caused by LTBI reactivation [31, 32]. Unlike the results in the present study, a previous study conducted in Korea reported that LTBI could be a risk factor for the development of TB [18]. At the time the previous study was conducted, LTBI management was not systematically implemented and only a low percentage of patients completed TB prophylaxis. In the present study, the percentage of LTBI patients receiving TB prophylaxis increased to $96 \%$. We believe that this difference may have significantly lowered the incidence of TB in LTBIpositive patients. As a result, the incidence of TB development in LTBI-positive patients was similar to that in LTBI-negative patients (Fig. 3). Moreover, a recent study reported the same 1-year incidence of TB in patients with IBD receiving anti-TNF therapy, regardless of LTBI status [33]. In another study, the expected reduction in the risk of TB development in the LTBI management group was $40-60 \%$ [34]. Thus, the results of the present study are in line with those of previous studies [33, 34] and confirm 


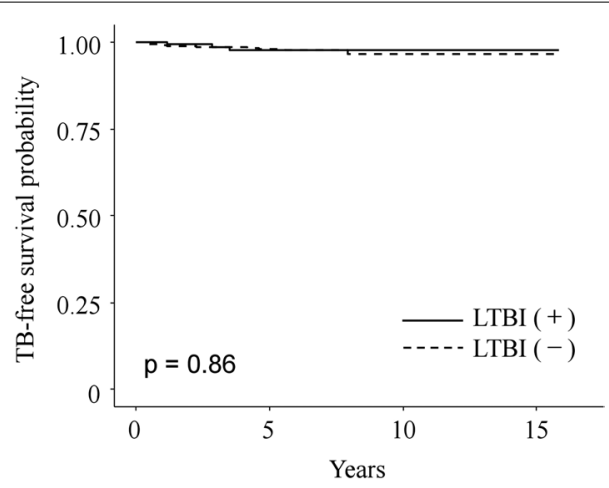

Fig. 3 Kaplan-Meier curve for the incidence of active tuberculosis in patients with IBD who underwent anti-TNF therapy, based on presence of latent tuberculosis infection (LTBI), as follows: LTBI-positive (solid line), LTBI-negative (dotted line)

that LTBI is no longer a risk factor for TB development in patients undergoing anti-TNF therapy who have also undergone active LTBI management.

Although active work-up and management of LTBI was performed in the present study, the onset of TB was not completely suppressed. Anti-TNF therapy can increase the risk of LTBI reactivation and increase susceptibility to primary TB and exogenous reinfection $[35,36]$. LTBI treatment can prevent TB development by LTBI reactivation, but it cannot prevent new active TB infection. Therefore, TB infection cannot be completely suppressed, even with active LTBI management; this is particularly important to consider in countries with a high TB burden. In addition, since the general indication for antiTNF therapy in patients with IBD is non-response to universal treatments such as steroids and/or immunomodulators, many patients with IBD are also given these drugs when anti-TNF therapy is administered, which can suppress the immune response and increase the chances of TB infection. One study found that immunosuppression by steroids and/or immunomodulators when starting anti-TNF therapy can lead to a low white blood cell count, which may be a risk factor for TB in patients undergoing anti-TNF therapy [18]. Finally, the diagnostic tests for LTBI are not completely accurate. The TST and IGRA are more likely to produce false negative results if the patients are taking drugs such as steroids or immunomodulators, or if they have an immunodeficiency [37-39]. In addition, IGRA has a relatively low sensitivity compared with its high specificity; therefore, the possibility of false-negative results should be considered. For these reasons, we believe that several cases of active TB development were identified at the beginning of anti-TNF therapy despite a negative LTBI test. To confirm that the tests were reliable, we checked the HR of TB development in the TST plus IGRA group and the IGRAonly group; there was no significant difference between the groups in this regard (HR 1.170, $p=0.765$ ). In immunosuppressed patients, researchers should be aware that the LTBI screening tests may be inaccurate and consider ways to increase the accuracy.

There were several limitations to the present study. First, LTBI evaluation was conducted in accordance with the Korean guidelines, so we did not consider several factors that can affect the test results. In particular, BCG vaccination can affect TST results and is mandatory in Korea [40], and immunosuppressants and various diseases can also affect result of TST and IGRA [37-39]. Second, although the age at which anti-TNF therapy was initiated correlated with the incidence rate of $\mathrm{TB}$, the time taken for TB to develop varies; therefore, care must be taken when interpreting this result. Third, clinicians tended to prefer infliximab as the first anti-TNF agent in the present study. For this reason, we could not fully explore the effects of other anti-TNF agents or multiple drugs on the development of TB and the identification of risk factors because they were given to a smaller proportion of patients. Lastly, it was difficult to confirm the accurate history of the other immunosuppressants (except anti-TNF agents) that had been administered at other medical centers. Consequently, we could not take into consideration other immunosuppressants that can influence the morbidity of TB. Further research with a larger population is needed in the future.

\section{Conclusions}

We confirmed that the incidence rate of TB increases linearly as the age at anti-TNF therapy initiation increases in patients with IBD. If anti-TNF therapy is administered to older patients, more rigorous monitoring for active TB development may be required. In addition, active LTBI screening and management may lower the incidence of TB development. Therefore, plans for monitoring TB development must be established, considering these characteristics.

\section{Abbreviations}

CD: Crohn's disease; IBD: Inflammatory bowel disease; IGRA: Interferon gamma release assay; LTBI: Latent tuberculosis infection; PPD: Purified protein derivative; TST: Tuberculin skin test; TB: Tuberculosis; TNF: Tumor necrosis factor; UC: Ulcerative colitis.

\section{Acknowledgements}

Not applicable.

\section{Authors' contributions}

$J Y L, K H O, H S H, K W K$, SWH, JHP, SWH, DHY, BDY, JSB, SJM, SKY, HSL, KWJ and SHP acquired the data. JYL, SWH, DHY, BDY, JSB, SJM, SKY, HSL, KWJ and SHP interpreted the data. JYL drafted the manuscript. BDY, SKY, KWJ and SHP critically revised the manuscript for important intellectual content. All authors read and approved the final manuscript. 


\section{Funding}

Not applicable.

\section{Availability of data and materials}

The data underlying this article cannot be shared publicly due to the privacy of individuals that participated in the study, but are available from the corresponding author (Sang Hyoung Park) on reasonable request.

\section{Declarations}

\section{Ethics approval and consent to participate}

The study was conducted in accordance with the Declaration of Helsinki, and all methods were carried out in accordance with relevant guidelines and regulations. The protocol was approved by the Institutional Review Board of Asan Medical Center (IRB No. 2018-1178). The requirement for informed consent was waived by the Institutional Review Board of Asan Medical Center due to the retrospective nature of the study.

\section{Consent for publication}

Not applicable.

\section{Competing interests}

All authors have no competing interest to declare.

\section{Author details}

${ }^{1}$ Department of Gastroenterology, Asan Medical Center, University of Ulsan College of Medicine, 88 Olympic-ro 43-gil, Songpa-gu, Seoul 05505, Korea. ${ }^{2}$ Department of Biochemistry, University of Ulsan College of Medicine, Asan Medical Center, 88 Olympic-ro 43-gil, Songpa-gu, Seoul 05505, Korea. ${ }^{3}$ Department of Pulmonary and Critical Care Medicine, Asan Medical Center, University of Ulsan College of Medicine, 88 Olympic-ro 43-gil, Songpa-gu, Seoul 05505, Korea.

Received: 18 June 2021 Accepted: 7 October 2021

Published online: 20 October 2021

\section{References}

1. Podolsky DK. Inflammatory bowel disease. N Engl J Med. 1991;325:928-37.

2. Katsanos KH, Papadakis KA. Inflammatory bowel disease: updates on molecular targets for biologics. Gut Liver. 2017;11:455.

3. Prideaux L, Kamm MA, De Cruz PP, Chan FK, Ng SC. Inflammatory bowel disease in Asia: a systematic review. J Gastroenterol Hepatol. 2012;27:1266-80.

4. Park SH, Kim Y-J, Rhee KH, Kim Y-H, Hong SN, Kim KH, et al. A 30-year trend analysis in the epidemiology of inflammatory bowel disease in the Songpa-Kangdong District of Seoul, Korea in 1986-2015. J Crohns Colitis. 2019:13:1410-7.

5. Yen $\mathrm{H}-\mathrm{H}$, Weng $\mathrm{M}-\mathrm{T}$, Tung $\mathrm{C}-\mathrm{C}$, Wang $\mathrm{Y}-\mathrm{T}$, Chang $\mathrm{YT}$, Chang $\mathrm{C}-\mathrm{H}$, et al. Epidemiological trend in inflammatory bowel disease in Taiwan from 2001 to 2015: a nationwide populationbased study. Intest Res. 2019;17:54.

6. Kaibullayeva J, Ualiyeva A, Oshibayeva A, Dushpanova A, Marshall JK. Prevalence and patient awareness of inflammatory bowel disease in Kazakhstan: a cross-sectional study. Intest Res. 2020;18:430.

7. Hong SW, Ye BD. The first step to unveil the epidemiology of inflammatory bowel disease in Central Asia. Intest Res. 2020;18:345.

8. Ordás I, Mould DR, Feagan BG, Sandborn WJ. Anti-TNF monoclonal antibodies in inflammatory bowel disease: pharmacokinetics-based dosing paradigms. Clin Pharmacol Ther. 2012;91:635-46.

9. Lee KM, Jeen YT, Cho JY, Lee CK, Koo JS, Park DI, et al. Efficacy, safety, and predictors of response to infliximab therapy for ulcerative colitis: AK orean multicenter retrospective study. J Gastroenterol Hepatol. 2013;28:1829-33.

10. Ye BD, Yang S-K, Shin SJ, Lee KM, Jang BI, Cheon JH, et al. Guidelines for the management of Crohn's disease. Korean J Gastroenterol. 2012;59:141-79.
11. Thorlund K, Druyts E, Mills EJ, Fedorak RN, Marshall JK. Adalimumab versus infliximab for the treatment of moderate to severe ulcerative colitis in adult patients naive to anti-TNF therapy: an indirect treatment comparison meta-analysis. J Crohns Colitis. 2014;8:571-81.

12. Kucharzik T, Ellul P, Greuter T, Rahier J-F, Verstockt B, Abreu C, et al. ECCO guidelines on the prevention, diagnosis, and management of infections in inflammatory bowel disease. J Crohns Colitis. 2021;15:879-913.

13. Hibi T, Kamae I, Pinton P, Ursos L, Iwakiri R, Hather G, et al. Efficacy of biologic therapies for biologic-naïve Japanese patients with moderately to severely active ulcerative colitis: a network meta-analysis. Intest Res. 2021;19:53-61.

14. Byun JM, Lee CK, Rhee SY, Kim H-J, Im JP, Park DI, et al. Risks for opportunistic tuberculosis infection in a cohort of 873 patients with inflammatory bowel disease receiving a tumor necrosis factor-a inhibitor. Scand J Gastroenterol. 2015;50:312-20.

15. Shim TS. Diagnosis and treatment of latent tuberculosis infection in patients with inflammatory bowel diseases due to initiation of antitumor necrosis factor therapy. Intest Res. 2014;12:12.

16. Horsburgh CR Jr, Rubin EJ. Latent tuberculosis infection in the United States. N Engl J Med. 2011;364:1441-8.

17. Ye BD, Travis S. Improving the quality of care for inflammatory bowel disease. Intest Res. 2019;17:45.

18. Kim ES, Am Song G, Cho KB, Park KS, Kim KO, Jang Bl, et al. Significant risk and associated factors of active tuberculosis infection in Korean patients with inflammatory bowel disease using anti-TNF agents. World J Gastroenterol: WJG. 2015;21:3308.

19. Park DI, Hisamatsu T, Chen M, Ng SC, Ooi CJ, Wei SC, et al. Asian Organization for Crohn's and Colitis and Asia Pacific Association of Gastroenterology consensus on tuberculosis infection in patients with inflammatory bowel disease receiving anti-tumor necrosis factor treatment. Part 2: management. J Gastroenterol Hepatol. 2018;33:30-6.

20. Kim JH, Yim J-J. Achievements in and challenges of tuberculosis control in South Korea. Emerg Infect Dis. 2015;21:1913.

21. Abitbol Y, Laharie D, Cosnes J, Allez M, Nancey S, Amiot A, et al. Negative screening does not rule out the risk of tuberculosis in patients with inflammatory bowel disease undergoing anti-TNF treatment: a descriptive study on the GETAID cohort. J Crohns Colitis. 2016;10:1179-85.

22. Agarwal A, Kedia S, Jain S, Gupta V, Bopanna S, Yadav DP, et al. High risk of tuberculosis during infliximab therapy despite tuberculosis screening in inflammatory bowel disease patients in India. Intest Res. 2018;16:588-98.

23. Korean Guidelines for Tuberculosis. 3rd edition. Korea Centers for Disease Control and Prevention. 2017.

24. Lee SH. Diagnosis and treatment of latent tuberculosis infection: the updated 2017 Korean guidelines. Korean J Med. 2018;93:509-17.

25. Kwak MS, Cha JM, Lee HH, Choi YS, Seo SI, Ko KJ, et al. Emerging trends of inflammatory bowel disease in South Korea: a nationwide populationbased study. J Gastroenterol Hepatol. 2019;34:1018-26.

26. Byng-Maddick R, Noursadeghi M. Does tuberculosis threaten our ageing populations? BMC Infect Dis. 2016;16:1-5.

27. Thomas TY, Rajagopalan S. Tuberculosis and aging: a global health problem. Clin Infect Dis. 2001:33:1034-9.

28. Yoshikawa TT. Tuberculosis in aging adults. J Am Geriatr Soc. 1992;40:178-87.

29. Yeon $\mathrm{JH}$, Seong $\mathrm{H}, \mathrm{Hur} H$, Park Y, Kim YA, Park YS, et al. Prevalence and risk factors of latent tuberculosis among Korean healthcare workers using whole-blood interferon- $\gamma$ release assay. Sci Rep. 2018;8:1-5.

30. Hong S, Kim H, Kim K, Han SJ, Ahn I, Ahn HS. Risk of incident Mycobacterium tuberculosis infection in patients with inflammatory bowel disease: a nationwide population-based study in South Korea. Aliment Pharmacol Ther. 2017;45:253-63.

31. Keane J, Gershon S, Wise RP, Mirabile-Levens E, Kasznica J, Schwieterman WD, et al. Tuberculosis associated with infliximab, a tumor necrosis factor a-neutralizing agent. N Engl J Med. 2001;345:1098-104.

32. Keane J. TNF-blocking agents and tuberculosis: new drugs illuminate an old topic. Rheumatology. 2005;44:714-20.

33. Kang J, Jeong DH, Han M, Yang S-K, Byeon J-S, Ye BD, et al. Incidence of active tuberculosis within one year after tumor necrosis factor inhibitor treatment according to latent tuberculosis infection status in patients with inflammatory bowel disease. J Korean Med Sci. 2018;33:e292. 
34. Erkens CG, Slump E, Verhagen M, Schimmel H, de Vries G, Cobelens F, et al. Monitoring latent tuberculosis infection diagnosis and management in the Netherlands. Eur Respir J. 2016;47:1492-501.

35. van Rie A, Warren R, Richardson M, Victor TC, Gie RP, Enarson DA, et al. Exogenous reinfection as a cause of recurrent tuberculosis after curative treatment. N Engl J Med. 1999;341:1174-9.

36. Chen D-Y, Shen G-H, Chen Y-M, Chen H-H, Hsieh C-W, Lan J-L. Biphasic emergence of active tuberculosis in rheumatoid arthritis patients receiving TNFa inhibitors: the utility of IFNy assay. Ann Rheum Dis. 2012;71:231-7

37. Debeuckelaere C, De Munter P, Van Bleyenbergh P, De Wever W, Van Assche $G$, Rutgeerts $P$, et al. Tuberculosis infection following anti-TNF therapy in inflammatory bowel disease, despite negative screening. J Crohns Colitis. 2014;8:550-7.

38. Wong SH, Gao Q, Tsoi KK, Wu WK, Tam L-S, Lee N, et al. Effect of immunosuppressive therapy on interferon $\gamma$ release assay for latent tuberculosis screening in patients with autoimmune diseases: a systematic review and meta-analysis. Thorax. 2016;71:64-72.

39. Alrajhi S, Germain P, Martel M, Lakatos P, Bessissow T, Al-Taweel T, et al Concordance between tuberculin skin test and interferon-gamma release assay for latent tuberculosis screening in inflammatory bowel disease. Intest Res. 2020;18:306-14.

40. Burl S, Adetifa UJ, Cox M, Touray E, Whittle H, McShane H, et al. The tuberculin skin test (TST) is affected by recent BCG vaccination but not by exposure to non-tuberculosis mycobacteria (NTM) during early life. PLOS ONE. 2010;5:e12287.

\section{Publisher's Note}

Springer Nature remains neutral with regard to jurisdictional claims in published maps and institutional affiliations.
Ready to submit your research? Choose BMC and benefit from:

- fast, convenient online submission

- thorough peer review by experienced researchers in your field

- rapid publication on acceptance

- support for research data, including large and complex data types

- gold Open Access which fosters wider collaboration and increased citations

- maximum visibility for your research: over $100 \mathrm{M}$ website views per year

At BMC, research is always in progress.

Learn more biomedcentral.com/submissions 